\title{
Longitudinal diffusion tensor imaging and neuropsychological correlates in traumatic brain injury patients
}

\author{
Kimberly D. Farbota ${ }^{1,2}$, Barbara B. Bendlin ${ }^{1,3}$, Andrew L. Alexander ${ }^{4}$, Howard A. Rowley ${ }^{5}$, \\ Robert J. Dempsey ${ }^{6}$ and Sterling C. Johnson ${ }^{1,3 *}$
}

${ }^{1}$ Geriatric Research and Education Clinical Center, William S. Middleton Memorial Veterans Hospital, Madison, WI, USA

${ }^{2}$ University of Wisconsin Neuroscience Training Program, Madison, WI, USA

${ }^{3}$ Department of Medicine, University of Wisconsin School of Medicine and Public Health, Madison, WI, USA

${ }^{4}$ Department of Medical Physics, University of Wisconsin School of Medicine and Public Health, Madison, WI, USA

${ }^{5}$ Department of Radiology, University of Wisconsin School of Medicine and Public Health, Madison, WI, USA

${ }^{6}$ Department of Neurological Surgery, University of Wisconsin School of Medicine and Public Health, Madison, WI, USA

\section{Edited by:}

Robin E. A. Green, University of

Toronto, Canada

Reviewed by:

Fernando Maestú, Complutense

University, Spain

David M. Schnyer, University of

Texas, USA

\section{*Correspondence:}

Sterling C. Johnson, Department of Medicine, William S. Middleton

Memorial VA Hospital, University of

Wisconsin, 2500 Overlook Terrace,

Madison, WI 53705, USA.

e-mail: scj@medicine.wisc.edu
Traumatic brain injury (TBI) often involves focal cortical injury and white matter (WM) damage that can be measured shortly after injury. Additionally, slowly evolving WM change can be observed but there is a paucity of research on the duration and spatial pattern of long-term changes several years post-injury. The current study utilized diffusion tensor imaging to identify regional WM changes in $12 \mathrm{TBI}$ patients and nine healthy controls at three time points over a four year period. Neuropsychological testing was also administered to each participant at each time point. Results indicate that TBI patients exhibit longitudinal changes to WM indexed by reductions in fractional anisotropy (FA) in the corpus callosum, as well as FA increases in bilateral regions of the superior longitudinal fasciculus (SLF) and portions of the optic radiation (OR). FA changes appear to be driven by changes in radial (not axial) diffusivity, suggesting that observed longitudinal FA changes may be related to changes in myelin rather than to axons. Neuropsychological correlations indicate that regional FA values in the corpus callosum and sagittal stratum (SS) correlate with performance on finger tapping and visuomotor speed tasks (respectively) in TBI patients, and that longitudinal increases in FA in the SS, SLF, and OR correlate with improved performance on the visuomotor speed (SS) task as well as a derived measure of cognitive control (SLF, OR). The results of this study showing progressive WM deterioration for several years post-injury contribute to a growing literature supporting the hypothesis that TBI should be viewed not as an isolated incident but as a prolonged disease state. The observations of long-term neurological and functional improvement provide evidence that some ameliorative change may be occurring concurrently with progressive degeneration.

Keywords: traumatic brain injury, diffusion tensor imaging, longitudinal, neuropsychology, recovery, DTI, TBI

\section{INTRODUCTION}

Traumatic brain injury (TBI) affects more than 1.4 million people every year in the United States (CDC, 2006). These injuries are the most common source of neurological impairment among young and middle-aged adults, and can produce long-term cognitive deficits that hinder patients' ability to function independently, lower their quality of life and increase the risk of developing comorbid neurological disorders (Anderson et al., 1995; Kiraly and Kiraly, 2007; Bombardier et al., 2010; Malec et al., 2010; Risdall and Menon, 2010; Sharp and Ham, 2011). Previous research has shown that the progression of structural pathology in the first year following injury includes decreased white matter (WM) integrity throughout the brain (Povlishock and Christman, 1995; Trivedi et al., 2007; Xu et al., 2007; Marquez de la Plata et al., 2008; Sidaros et al., 2008; Lin et al., 2010), but little is known about
WM changes that occur after this first year. Concomitant to slowly occurring atrophy and WM degradation, TBI patients typically demonstrate measurable cognitive and motor improvements in the first and subsequent years post-injury. This study aims to identify long-term patterns of WM change following TBI, as well as how variations in WM integrity correlate with both neuropsychological test performance and change in test performance over time (Levin, 2003; Staudt, 2010).

Diffusion tensor imaging (DTI) is sensitive to WM damage immediately following TBI and useful in monitoring longitudinal changes (Filippi et al., 2001; Xu et al., 2007; Bendlin et al., 2008). DTI, which is based on the principle that water molecule movement is restricted by barriers to diffusion that vary in the brain depending on tissue type or pathology, [for review see Le Bihan (1991)], is sensitive to changes in the microstructure of WM. 
Several studies have shown that DTI accurately detects damage in tissue that may appear normal when measured with conventional MRI (Arfanakis et al., 2002; Chan et al., 2003) and that DTI can be of clinical importance when tracing recovery (Filippi et al., 2001; Arfanakis et al., 2002; Field et al., 2003). These capabilities make DTI well suited for assessing WM damage caused by TBI and for tracking how WM changes progress longitudinally following injury.

The current study is an extension of a prior study conducted on this same cohort of TBI patients. The previous paper (Bendlin et al., 2008), included whole-brain DTI and volumetric analyses of patients at two months and one year post-injury. Results showed gray and WM alterations over time in TBI compared to control. Regions of reduced WM integrity included corpus callosum, forceps major and minor, anterior corona radiata, external capsule, cerebral peduncle, superior longitudinal fasciculus (SFL), uncinate fasciculus, and corticopontine tract. The current study extends the previous work with the addition of a third time point approximately four years post-injury. Furthermore, the current study includes axial and radial diffusivity analyses (which may allow inference concerning the cause of WM changes), and increases the power to detect group-wise effects by restricting analyses to the WM.

The primary metric used to assess WM integrity in this study is fractional anisotropy (FA). FA describes the extent to which a diffusion process is anisotropic or directionally constrained. In brain WM, higher FA is associated with greater WM integrity (Alexander et al., 2007). As noted above, we also employed two secondary metrics: axial diffusivity and radial diffusivity. Axial diffusivity refers to the movement of water along the principle axis of a WM tract. Animal studies have demonstrated that high axial diffusivity is associated with healthy axons, and low axial diffusivity is associated with axonal damage (Song et al., 2002, 2003). Radial diffusivity refers to the movement of water perpendicular to the principle axis of a WM tract. Animal studies have demonstrated that low radial diffusivity is associated with healthy myelin, and high radial diffusivity is associated with myelin damage (Wheeler-Kingshott and Cercignani, 2009). Investigating how these secondary metrics change over time in brain regions demonstrating longitudinal differences in FA can provide additional insight into the processes underlying WM change.

The first major goal of the current study was to characterize longitudinal changes in regional brain WM microstructure using DTI in conjunction with neuropsychological testing. Due to previous reports of volume loss and WM decline in the corpus callosum, as well as the emergent theory of TBI as the initiation of a disease state, we predicted that this structure would demonstrate continued decline throughout the duration of the study within the TBI group (Gale et al., 1995; Bigler et al., 1996; Kim et al., 2008; Kumar et al., 2010; Masel and DeWitt, 2010; Matsukawa et al., 2011; Ljungqvist et al., 2011). While decline in many regions is likely, patients typically continue to improve cognitively; thus we also sought to determine whether FA increases may occur in other regions, which may suggest consolidation or remodeling of WM tracts associated with recovery. Candidate regions include corticospinal tract regions (cerebral peduncle, internal capsule) and longitudinal tracts (superior and inferior longitudinal faciculi) due to previous research indicating improvements in certain diffusion metrics in a subset of these regions (Sidaros et al., 2008) and the presence of damage in these regions observed in crosssectional studies done close to the time of injury but not in those done several years post-TBI (Kraus et al., 2007; Bendlin et al., 2008).

The second major goal of this study was to identify correlations between FA and neuropsychological task performance cross-sectionally at each time point studied, as well as determine whether changes in task performance over time correlated with changes in FA longitudinally. Due to previous research indicating that higher corpus callosum FA is associated with better performance on manual motor tasks in brain injury patients, we predicted that scores on a fine motor finger tapping task employed in this study would correlate with FA in this region among TBI patients (Caeyenberghs et al., 2011a,b). Furthermore, we predicted that increases in FA within the longitudinal tracts would correlate with improved performance on the more complex neuropsychological tasks such as the Cognitive Oral Word Association Test (COWAT), or the cognitive component of the Trail Making Test. Finally, we expected patients who had sustained more severe injuries, as measured by the $24 \mathrm{~h}$ post-resuscitation Glasgow Coma Score (GCS), to demonstrate a greater degree of initial microstructural damage and longitudinal WM change than patients who sustained less severe injuries.

\section{METHODS TBI PATIENTS}

Forty-six TBI patients participated in an initial MRI scan, thirtysix returned for a second visit, and twenty returned for a third visit. DTI was acquired in sixteen individuals at all three time points (three were subsequently excluded due to excessive motion, and one more was excluded due to a second head injury sustained in a motor vehicle accident between visits 2 and 3). The mean age of the final group of twelve patients (ten males and two females) was $35.00 \pm 12.76$ years at the beginning of the study; mean education was $13.17 \pm 1.75$ years. The majority of the patients received acute treatment at the University of Wisconsin Hospital and Clinics level 1 trauma center and were referred from the departments of Neurosurgery, Trauma and/or Rehabilitation. The inclusion criteria for TBI consisted of involvement in a rapid impact injury to the brain (such as a motor vehicle accident or fall) causing a loss of consciousness. Evidence of brain injury included admittance for emergency medical attention following loss of consciousness in the field, a GCS score either at the emergency room (ER) or upon hospital admission of less than or equal to 13 , and a post-resuscitation GCS score of 5 or above. All patients had day of injury CT scans that were positive for visible brain injury. All TBI patients were less than three months post-injury at their first visit, and most were studied between eight and twelve weeks post-injury depending on their availability and other medical issues related to the injury. Exclusion criteria consisted of current major Axis I psychiatric disease or history of major medical condition (e.g., cancer, diabetes, or diagnosed neurological condition), as well as any previous diagnosis of substance dependence, or an undiagnosed pattern of behavior 
demonstrating longstanding maladaptive use of alcohol or other drugs. All patients gave informed written consent under a protocol approved by the University of Wisconsin Health Sciences Institutional Review Board.

\section{HEALTHY CONTROLS}

Thirty-six control participants were recruited from the community and from the University of Wisconsin Madison campus via advertisement. Twenty of these participants returned for two additional visits. Acquisition errors resulted in the loss of four participants who did not have adequate DTI at all three time points; and in seven cases DTI was not acquired at one of the visits due to time constraints; DTI scans acquired from nine participants at all three time points were used in the final analysis. The mean age of the final group of nine healthy controls (four males, five females) was $31.44 \pm 12.38$ years at the beginning of the study; education was $14.77 \pm 2.22$ years. Exclusion criteria were identical to the TBI group (with the exception of head injury also being exclusionary for controls). MR scanning of control participants occurred on approximately the same schedule as that of TBI patients. All participants gave informed written consent under a protocol approved by the University of Wisconsin Health Sciences Institutional Review Board.

\section{PROCEDURES}

Volunteers participated in three testing sessions, each consisting of MR imaging and neuropsychological testing. TBI patients were tested at three visits, Visit 1, acquired approximately two months (mean $=63$ days) post-injury (ranging from 28 to 81 days), Visit 2 , approximately one year (mean $=318$ days) after Visit 1 (ranging from 226 to 381 days) and Visit 3, approximately three years $($ mean $=1187$ days) after Visit 2 (ranging from 956 to 1651 days). Controls also participated in three visits with approximately one year $($ mean $=286$ days) between Visit 1 and 2 (ranging from 74 to 374 days) and three years (mean $=1096$ days) between Visit 2 and 3 (ranging from 778 to 2011 days).

\section{NEUROPSYCHOLOGICAL EXAMINATION}

On the day of each scan, a neuropsychological battery that included: COWAT, WRAT-III (Wide Range Achievement Testreading subtest, an approximation of pre-morbid intelligence), Finger Tapping (during which participants tapped a lever for $10 \mathrm{~s}$ as fast as possible for three trials and the average was used), Digit Span (a measure of working memory) and Trail Making Tests A (a visual-motor speed task) and B (a combination task requiring both visual-motor skill and rapid cognitive set shifting) was administered to each participant. These tests were selected based on previous research in our laboratory suggesting their probable relevance to TBI induced behavioral changes. Some of our analyses also included Trails B cognitive component scores which were calculated by subtracting each subject's Trails A score (visuomotor) from his or her Trails B score (visuomotor and cognitive) to isolate the cognitive component of the Trails B task. Statistical analysis of neuropsychological test results was performed as follows: for each test, a general linear model repeated measures test was carried out in SPSS 20.0. From these models, main effects of group and time as well as a group by time interaction were derived. Simple effects analyses were performed by using independent samples one-tailed $t$-tests to assess between-groups differences in task performance at each time point, and by using paired samples one-tailed $t$-tests to assess within groups changes in task performance across time points.

\section{MAGNETIC RESONANCE IMAGING}

All participants underwent magnetic resonance on a General Electric 3.0 T (Waukesha, WI) MRI system with a quadrature birdcage head coil. Structural scans included an axial T1-weighted inversion recovery-prepped spoiled gradient echo scan (inversion time $=600 \mathrm{~ms}$, repetition time (TR)/echo time (TE)/flip angle $=9 \mathrm{~ms} / 1.8 \mathrm{~ms} / 20^{\circ}$; acquisition matrix $=256 \times 192$ interpolated to $256 \times 256$; field of view $(\mathrm{FOV})=240 \mathrm{~mm}$; and 124 slices $1.2 \mathrm{~mm}$ thick). Diffusion tensor imaging was performed using a cardiac-gated, diffusion-weighted sequence with the following parameters: 12 directions with diffusion weighting of $1114 \mathrm{~s} / \mathrm{mm}^{2}$ and a non-diffusion-weighted reference image (B0); $\mathrm{TR}=10-15 \mathrm{~s}$; TE $=78.2 \mathrm{~ms}$; number of averages: 3 ; acquisition matrix $=120 \times 120$ interpolated to $256 \times 256$; FOV $=240 \mathrm{~mm}$; 39 contiguous $3 \mathrm{~mm}$ thick axial slices. The scan resulted in $0.9375 \times 0.9375 \times 3 \mathrm{~mm}$ voxels. Prior to the diffusion-weighted scan, high order shimming was performed to minimize EPI distortions. A neuroradiologist (HR) reviewed all structural MRI images to identify the location and extent of lesions associated with the TBI and to identify non-injury related brain abnormalities that might exclude participants from the statistical analyses. Additionally, a high resolution 2D axial $\mathrm{T}^{*}$ gradient echo sequence sensitive to both DAI and contusions was collected for evaluation by a neuroradiologist who confirmed the presence of brain injury. Imaging parameters were as follows: gradient echo read-out with $\mathrm{TR}=325 \mathrm{~ms}$, $\mathrm{TE}=20 \mathrm{~ms}$; flip angle $=15^{\circ}$; acquisition matrix $=256 \times 192$ interpolated to $256 \times 256 ; \mathrm{FOV}=240 \mathrm{~mm} ; 225 \mathrm{~mm}$ thick axial slices, with a $1 \mathrm{~mm}$ skip between slices; and receiver bandwidth $= \pm 15.83 \mathrm{kHz}$.

\section{DIFFUSION TENSOR IMAGE PROCESSING}

Image distortions in the DTI-data caused by eddy currents were corrected using a 2D affine co-registration function, align linear, in the Automated Image Registration software package (http:// www.loni.ucla.edu/Software/AIR). Non-linear image distortion from static field (B0) inhomogeneities was corrected using the acquired field map and implemented in the prelude (Phase Region Expanding Labeller for Unwrapping Discrete Estimates) and fugue (FMRIB's Utility for Geometrically Unwarping EPIs) tools from the FSL software suite (Smith et al., 2004). After distortion corrections, 3D maps of the diffusion tensor and derived measures, FA, axial diffusivity (determined using the principle eigenvalue, L1) and radial diffusivity (determined using the average of the secondary and tertiary eigenvalues, L2 and L3), were calculated. For each subject, the FA, radial diffusivity, and axial diffusivity maps from Visits 2 and 3 were then co-registered to the corresponding maps from Visit 1 using flirt (FSL) 12-parameter affine co-registration. Normalization was then performed using fNIRT in FSL. Normalization of the Visit 1 maps to the FSL FMRIB58_FA_1 mm template was performed 
for each subject. The transformation derived from this normalization was then applied to the co-registered maps acquired during Visits 2 and 3. The maps from each time point for each measure were visually checked for alignment to each other and the template.

\section{STATISTICAL ANALYSIS}

Primary statistical analyses were performed on FA maps using factorial ANOVA statistical modules in SPM8. Gender was used as a covariate in all analyses. Because this study focused only on WM change, all SPM analyses were restricted to regions within a WM mask to increase our power to detect longitudinal change. The WM mask was created by applying a threshold of 0.5 (Figure 1) to the mean FA map of all subjects, and then binarizing the resulting image using fslmaths (FMRIB Software Library). To determine whether there were overall group differences in longitudinal FA change, we tested for an interaction between group and time within the factorial model. The hypothesis that there would be longitudinal change within the TBI group was tested using simple effects analyses, also within the factorial model framework.

Secondary statistical analyses using axial and radial diffusivity maps were also performed using factorial ANOVA statistical modules in SPM8. Because these analyses were employed to further examine the causes of FA change, axial, and radial diffusivity analyses were restricted to regions where significant FA results were observed. We hypothesized that increases in FA would be driven by decreased radial diffusivity and/or increased
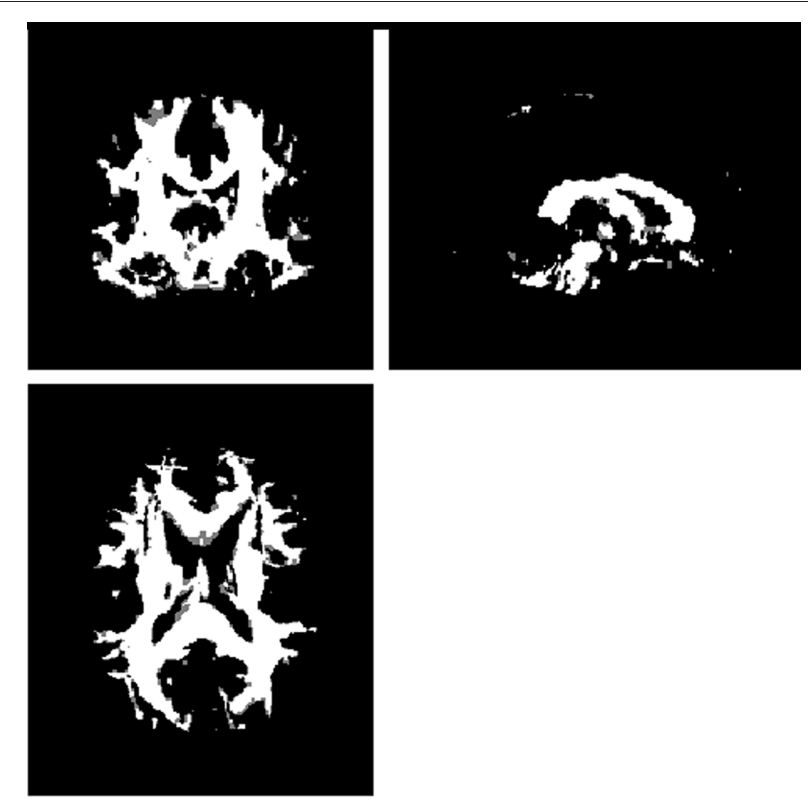

FIGURE 1 | Because this study focused only on white matter change, all SPM analyses were restricted to regions within a white matter mask to increase our power to detect longitudinal change. The white matter mask was created by applying a threshold of 0.5 to the mean FA map of all subjects, and then binarizing the resulting image. This figure shows the white matter mask in three orthogonal directions. The left side of the mask is patient left and the right is patient right. axial diffusivity, and also conversely that decreases in FA would be driven by increased radial diffusivity and/or increased axial diffusivity.

Correlations with neuropsychological test scores were assessed using linear regression implemented in SPM8, where test scores were independent variables and FA maps were the dependent variables. We hypothesized that we would see positive regional correlations between FA and task performance. In addition to direct correlations between neuropsychological test scores and FA values, we also tested hypotheses concerning how changes in FA and changes in neuropsychological performance might correlate. In these cases, differences in neuropsychological test scores between two time points were calculated for each subject, as were differences in FA during the same interval. FA differences were determined by subtracting the later maps from the earlier maps for each subject. Correlations between FA change maps and changes in neuropsychological test scores were also assessed using linear regression implemented in SPM8, where changes in test scores were independent variables and FA change maps were the dependent variables. All correlation analyses of neuropsychological measures were limited to regions within the WM mask used in general FA analyses.

\section{STATISTICAL THRESHOLD}

A voxel-level threshold of $\alpha=0.001$ (uncorrected) was used for all contrasts. Multiple-comparison correction for FA analyses was performed using estimates from a Monte-Carlo simulation performed with AlphaSim to achieve a corrected cluster-level threshold of $\alpha=0.05$ (Forman et al., 1995). The Monte-Carlo simulation determined based on randomly computed images that a cluster with 581 voxels with the same dimensions, voxel probability threshold, and smoothness parameters as FA images inputted for analysis would be unlikely (at the $\alpha=0.05$ clusterlevel corrected threshold) to be significant only by chance. Thus, a cluster-level threshold of 581 voxels was used in all FA analyses. For secondary factorial analyses of axial and radial diffusivity and regression analyses used to test neuropsychological correlations cluster-levels were determined individually for each SPM at the significance levels noted above.

\section{RESULTS}

\section{DEMOGRAPHIC AND BEHAVIORAL RESULTS}

There were no significant differences in age $(t=-1.131, \mathrm{df}=19$, two-tailed $p=0.272)$ or years of education $(t=-1.861$, $\mathrm{df}=19$, two-tailed $p=0.078)$ between the TBI and control groups. There were significantly more males in the TBI group compared to the control group $\left(\chi_{(1,12)}^{2}=4.535, p=0.033\right)$. Demographic results are shown in Table 1. Repeated measures analyses of neuropsychological test results indicated a main effect of group in the DSPAN, Trails B, and COWAT tasks, as well as a group by time interaction for the COWAT task (Table 2). Simple effects analyses of neuropsychological tests performance indicated that TBI patients' performance was significantly worse than that of controls on Digit Span, Trails A and B at Visits 1,2 , and 3. TBI patients also differed from controls on the COWAT at Visits 1 and 2. No significant group differences were seen in WRAT-III or Finger Tapping. TBI patients demonstrated 
Table 1 | Individual level subject demographics for patients and controls and select patient injury characteristics.

\begin{tabular}{|c|c|c|c|c|c|c|c|}
\hline Subject & Age & Education & Sex & GCSadmit & GCS24 & Hrs15 & Injury notes \\
\hline \multicolumn{8}{|l|}{ PATIENTS } \\
\hline 1 & 19 & 12 & M & 3T & 7 & 334 & DAl \\
\hline 3 & 19 & 14 & $\mathrm{~F}$ & $3 T$ & 11 & 91 & Subarachnoid hemorrhage, subdural hematoma \\
\hline 4 & 24 & 14 & M & 3 & 7 & 662 & DAl, contusions, epidural hematoma, subarachnoid hemorrhage \\
\hline 7 & 29 & 13 & M & 3 & 14 & 179 & DAl, contusions, skull fracture \\
\hline 8 & 52 & 16 & $\mathrm{~F}$ & 11 & 13 & 97 & Skull fracture, subarachnoid hemorrhage, subdural hematoma, \\
\hline 9 & 51 & 12 & M & 7 & 7 & 726 & Depressed skull fracture, subdural and epidural hematomas \\
\hline 10 & 19 & 13 & M & 3T & 5 & 49 & Extensive hemorrhages, CC shearing \\
\hline 11 & 37 & 14 & M & 9 & 7 & 116 & DAI, subarachnoid hemorrhage, subdural hematoma, shearing \\
\hline 13 & 22 & 15 & M & & & & \\
\hline 14 & 29 & 13 & $\mathrm{~F}$ & & & & \\
\hline 15 & 27 & 16 & $\mathrm{~F}$ & & & & \\
\hline 16 & 25 & 12 & $\mathrm{~F}$ & & & & \\
\hline 17 & 19 & 16 & $\mathrm{~F}$ & & & & \\
\hline 18 & 36 & 16 & M & & & & \\
\hline 19 & 24 & 13 & M & & & & \\
\hline 20 & 51 & 13 & M & & & & \\
\hline Mean (SD) & $29.2(9.7)$ & $14.8(2.2)$ & $44 \% \mathrm{M}$ & & & & \\
\hline$p$ & 0.272 & 0.078 & 0.033 & & & & \\
\hline
\end{tabular}

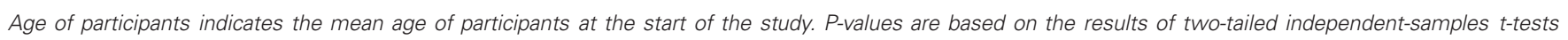
for age and education and a chi-squares test for gender proportions. Abbreviations are as follows: GCSadmit = Glasgow Coma Scale score at hospital admission, GCS24 = Glasgow Coma Scale score $24 \mathrm{~h}$ post-injury, $15 \mathrm{~h}=$ Number of hours before patient reached a GCS score of 15, DAl= Diffuse axonal injury, CC $=$ corpus callosum, $T=$ Patient was intubated at the time of GCS assessment.

significant improvements on the DSPAN, Trails A, B, and COWAT tests between Visit 1 and 2, and also showed significant improvement on the Trails A, B and COWAT tests between Visit 1 and 3. Controls demonstrated significant improvement on the Trails A and B tasks between Visit 1 and 2. All neurological test results are shown in Table 2.

\section{DTI RESULTS}

The FA factorial analysis revealed a group by time interaction in the genu of the corpus callosum (Figure 2). Analyses of the secondary metrics (using separate factorial models for axial diffusivity and radial diffusivity maps) demonstrated that there was also a group by time interaction in this region in the radial diffusivity model, but not the axial diffusivity model (Figure 2). Together these analyses indicate that the group by time interaction observed in the genu in the FA analysis was driven by changes in radial (not axial) diffusivity.

A main effect of time was observed throughout the corpus callosum in the primary FA analysis (Figure 3). A corresponding main effect of time was observed in this region in the radial, but not the axial, diffusivity analysis (Figure 3). A main effect of group was observed in WM tracts throughout the brain, including the cerebral peduncle, inferior and superior longitudinal fascicule (ILF and SLF), internal and external capsule, inferior fronto-occipital fasciculus, sagittal stratum (SS), corpus callosum, fornix, optic radiations (ORs), thalamic radiations, uncinate fasciculus, and corona radiata (Figure 4). Axial and radial diffusivity analyses revealed a main effect of group in genu, fornix, and ILF in the axial model and a main effect of group throughout the corpus callosum, as well as in the fornix, ILF, ORs, and thalamic radiations in the radial model (Figure 4).

Simple effects analyses within the FA factorial model demonstrated that TBI subjects exhibited a significant decrease in FA throughout the corpus callosum between the first and third visits (Figure 5A). We hypothesized that this change would be driven either by a decrease in axial diffusivity, an increase in radial diffusivity, or some combination of the two. Analyses of the secondary metrics limited to this corpus callosum region were used to test this hypothesis. Results showed that TBI subjects did not demonstrate any decreases in axial diffusivity in this region, while they did exhibit significant increases in radial diffusivity in both the genu and isthmus of the corpus callosum (Figure 5A). Controls did not demonstrate significant longitudinal FA decreases in any brain regions. 
Table 2 | Neuropsychological test results.

\begin{tabular}{|c|c|c|c|}
\hline Task & $\begin{array}{l}\text { Time } 1 \\
n, \text { Mean (SD) }\end{array}$ & $\begin{array}{l}\text { Time } 2 \\
n \text {, Mean (SD) }\end{array}$ & $\begin{array}{l}\text { Time } 3 \\
n, \text { Mean (SD) }\end{array}$ \\
\hline \multicolumn{4}{|c|}{ TBI GROUP RESULTS } \\
\hline WRAT-III & $12, \mathbf{4 4 . 7}(6.5)$ & $12,44.5(12.3)$ & $11,45.6(5.7)$ \\
\hline DSPAN $^{\mathrm{a}}$ & $12, \mathbf{1 4 . 3}^{* * * \dagger}(3.6)$ & $12, \mathbf{1 5 . 6}^{*}(3.7)$ & $11, \mathbf{1 5 . 6}^{*}(4.6)$ \\
\hline Trails A & $12, \mathbf{4 2 . 0}^{* \dagger}(21.5)$ & $12, \mathbf{3 0 . 8}^{*}(6.4)$ & $12, \mathbf{2 8 . 7}^{* * * \dagger}(7.1)$ \\
\hline Trails $B^{a}$ & $11, \mathbf{1 0 1 . 3}^{* \dagger}(29.0)$ & $12, \mathbf{7 8 . 6}^{*}(20.9)$ & $12, \mathbf{6 8 . 8}^{* \dagger}(36.4)$ \\
\hline COWAT ${ }^{\mathrm{b}}$ & 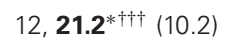 & $12, \mathbf{3 3 . 8 *}(10.6)$ & $12, \mathbf{3 9 . 6}^{\dagger \dagger}(12.4)$ \\
\hline FT & $10,44.1(14.7)$ & $12,45.9(7.6)$ & $12,45.7(6.9$ \\
\hline \multicolumn{4}{|c|}{ CONTROL GROUP RESULTS } \\
\hline WRAT-III & $8, \mathbf{5 0 . 0}(5.1)$ & $7, \mathbf{5 0 . 0}(6.1)$ & $9,55.7(5.2)$ \\
\hline DSPAN & $8,20.1(3.4)$ & 7, $20.6(3.9)$ & $9,20.4(5.2)$ \\
\hline Trails A & $8,25.1(6.9)$ & $7, \mathbf{2 4 . 6}^{\dagger \dagger}(5.4)$ & $9, \mathbf{2 0 . 3}(4.5)$ \\
\hline Trails B & $8, \mathbf{5 5 . 0}(25.6)$ & $7, \mathbf{5 1 . 5}^{\dagger}(20.4)$ & $9,38.6(7.1)$ \\
\hline COWAT & $8,41.8(7.8)$ & 7, 44.6 (8.9) & $9,45.9(11.9)$ \\
\hline FT & $6,45.6(10.8)$ & $7,48.9(6.9)$ & $9,48.3(6.8)$ \\
\hline
\end{tabular}

Test abbreviations are as follows: WRAT-III = Wide Range Achievement Test (reading subtest), DSPAN $=$ Digit Span Test, Trails $A=$ Trail Making Test $A$ (motor), Trails $B=$ Trail Making Test $B$ (motor and cognitive), COWAT = Cognitive Oral Word Association Test, FT DOM = Dominant Hand Finger Tapping Test. Means are of raw scores. Repeated measures analyses based on general linear models were carried out for each neuropsychological test and significant results $(p<0.05)$ are noted next to task names in the following manner: ${ }^{a}$ indicates a main effect of group and ${ }^{b}$ indicates a group by time interaction. None of the models demonstrated a significant effect of time. Between-groups differences were calculated using independent samples one-tailed t-tests, and significance levels are denoted as follows: ${ }^{*} p<0.05,{ }^{* *} p<0.01,{ }^{* * *} p<0.001$. Between groups differences are noted by the patient average scores only. Within-groups differences were calculated using paired samples one-tailed t-tests, and significance levels are denoted as follows: ${ }^{\dagger} p<0.05,{ }^{\dagger \dagger} p<0.01,{ }^{\dagger \dagger} p<0.001$. Differences between time 1 and 2 are noted by the first time point average, differences between time 2 and 3 are noted by the second time point average, and differences between time 1 and 3 are noted by the third time point average.

In order to determine whether the decrease in FA in the corpus callosum within the TBI group between the first and third visits was driven primarily by early changes during the first interval or late changes during the second interval, each interval was assessed individually within the FA factorial model. These secondary analyses were limited to the corpus callosum region where a change had been observed between the first and third time points. Results demonstrated that significant changes could be seen in the genu and body of the corpus callosum during the first interval (Figure 5B), but no significant clusters were identified during the second interval. Axial and radial diffusivity analyses within this region indicated that an increase in radial diffusivity was present in the genu during the first interval among TBI subjects (Figure 5B), however, no accompanying decrease in axial diffusivity was observed.

Simple effects analyses within the FA factorial model also demonstrated that TBI subjects exhibited significant FA increases in bilateral regions of the SLF as well as in an OR region on the left side of the brain between the first and third visits (Figure 6). Again, we hypothesized that changes observed among TBI patients would be driven either by decreases in axial diffusivity, increases in radial diffusivity, or some combination of the two. Analyses of the secondary metrics limited to these regions were used to test this hypothesis. Results showed that TBI subjects did not demonstrate any increases in axial diffusivity in these regions between the first and third visits, however, significant decreases in radial diffusivity were observed in all regions tested (Figure 6). Controls did not exhibit significant longitudinal FA increases in any brain regions.

In order to determine whether the increases in FA in the bilateral SLF and left OR within the TBI group between the first and third visits were driven primarily by early changes during the first interval or late changes during the second interval, each interval was assessed individually within the FA factorial model. These secondary FA increase analyses were limited to the regions where a change had been observed between the first and third time points. No significant clusters were identified during either the first or second intervals individually, suggesting that the observed change occurred gradually over the four year study duration.

Between-groups simple effects analyses demonstrated that the TBI group had reduced FA compared to the control group in the cerebral peduncle, ILF, SLF, internal and external capsule, inferior fronto-occipital fasciculus, SS, corpus callosum, fornix, ORs, thalamic radiations, uncinate fasciculus, and corona radiata at all three visits. There were no regions in which the TBI group had greater FA than the control group at any of the three time points. We hypothesized that FA reductions would be driven by a combination of reduced axial and increased radial diffusivity. Secondary analyses confirmed this hypothesis, demonstrating that the TBI group had reduced axial diffusivity compared to controls in parts of the cerebral peduncle, external capsule, internal capsule, OR, fornix, SLF, and ILF at all three time points. Increased radial diffusivity among TBI patients was observed in all regions in which group differences in FA were observed at all three time points.

\section{NEUROPSYCHOLOGICAL CORRELATIONS}

Contrary to our hypothesis, we did not observe any correlations between an individual's GCS score and regional WM FA at any of the three time points. Of the seven neuropsychological measures tested for correlation with FA, two tests, Trails A and dominant hand finger tapping, were significantly correlated with regional FA values among the TBI subjects. No significant correlations were observed between FA and neuropsychological test performance among control subjects. Finger tapping scores correlated positively with FA in the splenium of the corpus callosum at the second time point (Figure 7). A lowered statistical threshold $\alpha=0.01$ enabled observation of smaller splenium clusters when the equivalent correlational tests were run for the first and third time points, however, only the second time point result surpassed significance levels employed in this study. Trails A performance correlated positively with FA in bilateral regions of the SS at the first time point, and a unilateral region in the left SS at the second time point (Figure 8A).

Correlations between FA changes and changes in neuropsychological measures over time were also significant for two neuropsychological measures, Trails A and B cognitive component. No equivalent correlations were observed among controls for any measure. There was a positive correlation between change in the 

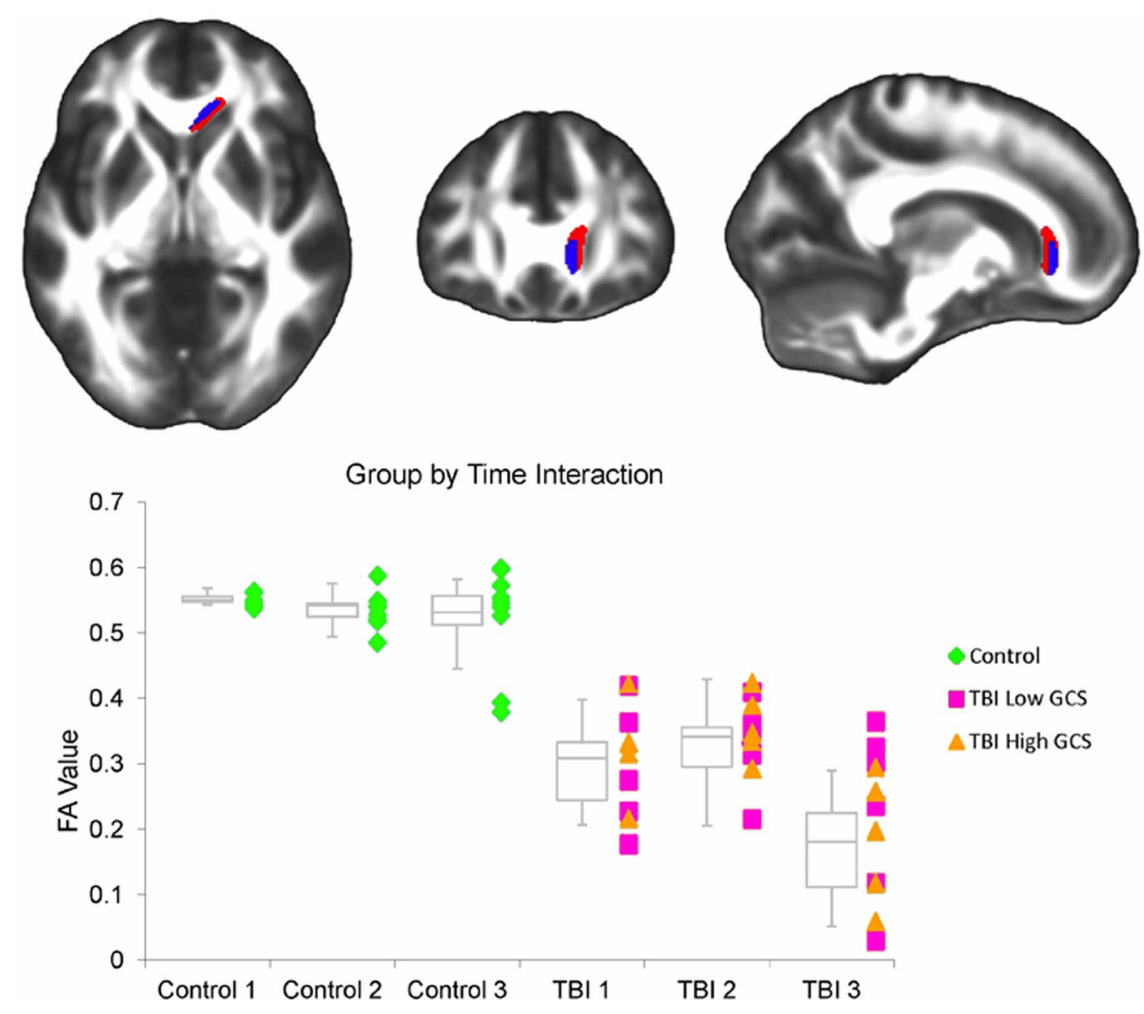

FIGURE 2 | (Top) A significant interaction between group and interval was observed within the FA factorial model. There were inter-interval dissimilarities in group-wise patterns of FA change in the genu of the corpus callosum (red). Axial and radial diffusivity analyses were restricted to regions demonstrating an FA effect. Within this region, a significant interaction was also observed in the radial diffusivity factorial model (overlapping blue region), but not the axial diffusivity factorial model. The left side of the statistical map is patient left and right is patient right. (Bottom) This graph shows the average FA value for each subject at each of the three time points within the corpus callosum cluster demonstrating an FA effect. Controls are marked as green diamonds, TBI patients with a $24 \mathrm{~h} \mathrm{GCS} \mathrm{score} \mathrm{of}$ 7 or lower are marked as pink squares, and patients with a $24 \mathrm{~h}$ GCS score of 10 or higher are marked as orange triangles. Box plots indicate the 75th percentile, median, and 25th percentile FA values of each group at each time point, and whiskers indicate 1.5 times inter-quartile ranges.
Trails A score between the first and third time points and change in FA over the same duration (where an increase in FA was associated with a reduction in time to complete Trails A) in the right SS (Figure 8B). No significant correlations were observed between change in Trails A score and change in FA during either subinterval of the study (from time one to time two or from time two to time three). A positive correlation between FA change and change in the Trails B cognitive component between time one and time three was observed in the left superior SLF and the right OR (Figure 9). Analysis of this correlation during each of the sub-intervals did not reveal any regions of significant clusters between the first and second time point, but a significant cluster was observed in the right posterior SLF between the second and third time points (Figure 9).

\section{DISCUSSION}

Longitudinal brain changes following TBI are sparsely documented. In this study we examined TBI patients over a period of four years and found that rather than showing a circumscribed period of brain degeneration following injury, TBI involves a protracted period of brain change that continues for several years. The results of this study suggest that studying alterations in brain
WM may provide clues to neuropsychological function following TBI, and potentially inform upon the clinical course of patients following injury.

In our study, we found significant effects in the corpus callosum, which is commonly injured in TBI. The group by time interaction observed in the FA factorial model combined with the simple effects analyses indicates that the TBI subjects demonstrated a significantly greater reduction in FA in the genu of the corpus callosum during the first year post-injury than during the subsequent three years of the total followup period. This result is commensurate with previous work on a different subset of individuals in this cohort indicating FA reductions in this region during the first year post-injury (Bendlin et al., 2008), as well as other previous studies that have also found longitudinal WM decline in this region during the first year (Xu et al., 2007; Wu et al., 2010). Our results also demonstrated that the observed FA effect was driven by increased radial, rather than decreased axial, diffusivity, which is consistent with previous observations (Sidaros et al., 2008; Kumar et al., 2010). Other recent research has indicated that initial injury to the genu is highly predictive of patient outcome (in terms of general disability), however, we did not 

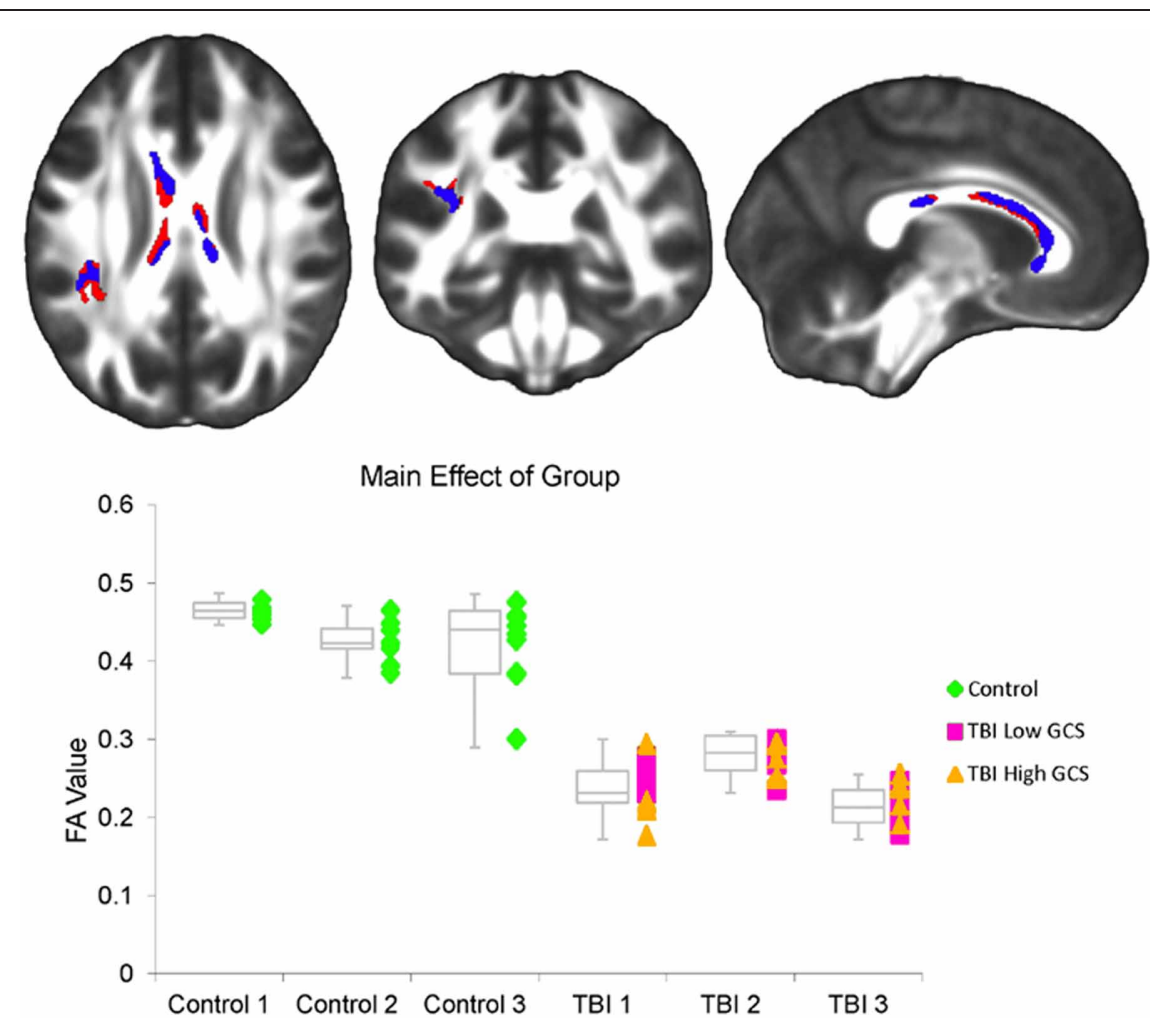

Control
TBI Low GCS
$\triangle$ TBI High GCS

FIGURE 3 | (Top) A significant effect of time was observed throughout the corpus callosum as well as in the left SLF within the FA factorial model (red). Axial and radial diffusivity analyses were restricted to regions demonstrating an FA effect. Within this region, a significant effect of time was also observed within the radial diffusivity factorial model (overlapping blue region), but not the axial diffusivity factorial model. The left side of the statistical map is patient left and right is patient right. (Bottom) This graph shows the average
FA value for each subject at each of the three time points within the corpus callosum cluster demonstrating an FA effect. Controls are marked as green diamonds, TBI patients with a $24 \mathrm{~h}$ GCS score of 7 or lower are marked as pink squares, and patients with a $24 \mathrm{~h}$ GCS score of 10 or higher are marked as orange triangles. Box plots indicate the 75th percentile, median, and 25th percentile FA values of each group at each time point, and whiskers indicate 1.5 times inter-quartile ranges. observe correlations between FA change in this region over time and change in neuropsychological task performance (Matsukawa et al., 2011).

The main effect of time combined with simple effects analyses indicate that TBI patients experienced a continued decline in FA throughout the corpus callosum that continued throughout the duration of the four year period studied, and that this decrease was driven by increases in radial diffusivity. It has been suggested that initial tearing, shearing, and misalignment of axons initiates an inflammatory cascade that leads to further WM damage, myelin loss, and gliosis, and we expect that these processes were critical to the gradual, long-term FA reductions observed here (Povlishock, 2000).

The main effect of group combined with between-groups simple effects analyses indicate that TBI subjects exhibited reduced FA in several major tracts, that this difference was persistent for at least four years post-injury, and that a combination of reduced axial and increased radial diffusivity gave rise to these FA differences. These results collectively demonstrate that in several major tracts there is reduced directional coherence of WM among our TBI subjects that is both widespread and long-lasting. These between-groups differences are consistent with cross-sectional observations (Chan et al., 2003; Nakayama et al., 2006; Kiraly and
Kiraly, 2007; Xu et al., 2007; Bendlin et al., 2008; Sidaros et al., 2008; Wang et al., 2011).

Our simple effects results also demonstrate that TBI subjects demonstrated increases in FA in the SLF bilaterally as well as in a portion of the OR during the course of the study, potentially signifying improvement of WM integrity or alternatively showing loss of crossing fibers in these brain regions. No previous study that we are aware of has demonstrated longitudinal increases in FA among TBI patients. This is likely because no previous study that we are aware of followed patients for four years as the current study did, and the FA increases we observed appear to have taken place gradually during the four year study duration. Previous research has, however, identified apparent improvements in FA in animal models of TBI (Rubovitch et al., 2011), and in either axial or radial diffusivity among human TBI patients (Sidaros et al., 2008; Kumar et al., 2010), indicating that our present finding is potentially replicable. This evidence of subtle neurological recovery merits further investigation, particularly in clinical settings. The secondary result indicating that this increase in FA was driven by a longitudinal decrease in radial diffusivity (rather than an increase in axial diffusivity) could suggest improved myelin integrity within the tract, however, it is equally plausible that progressive loss of damaged axons that is observed in animal models 

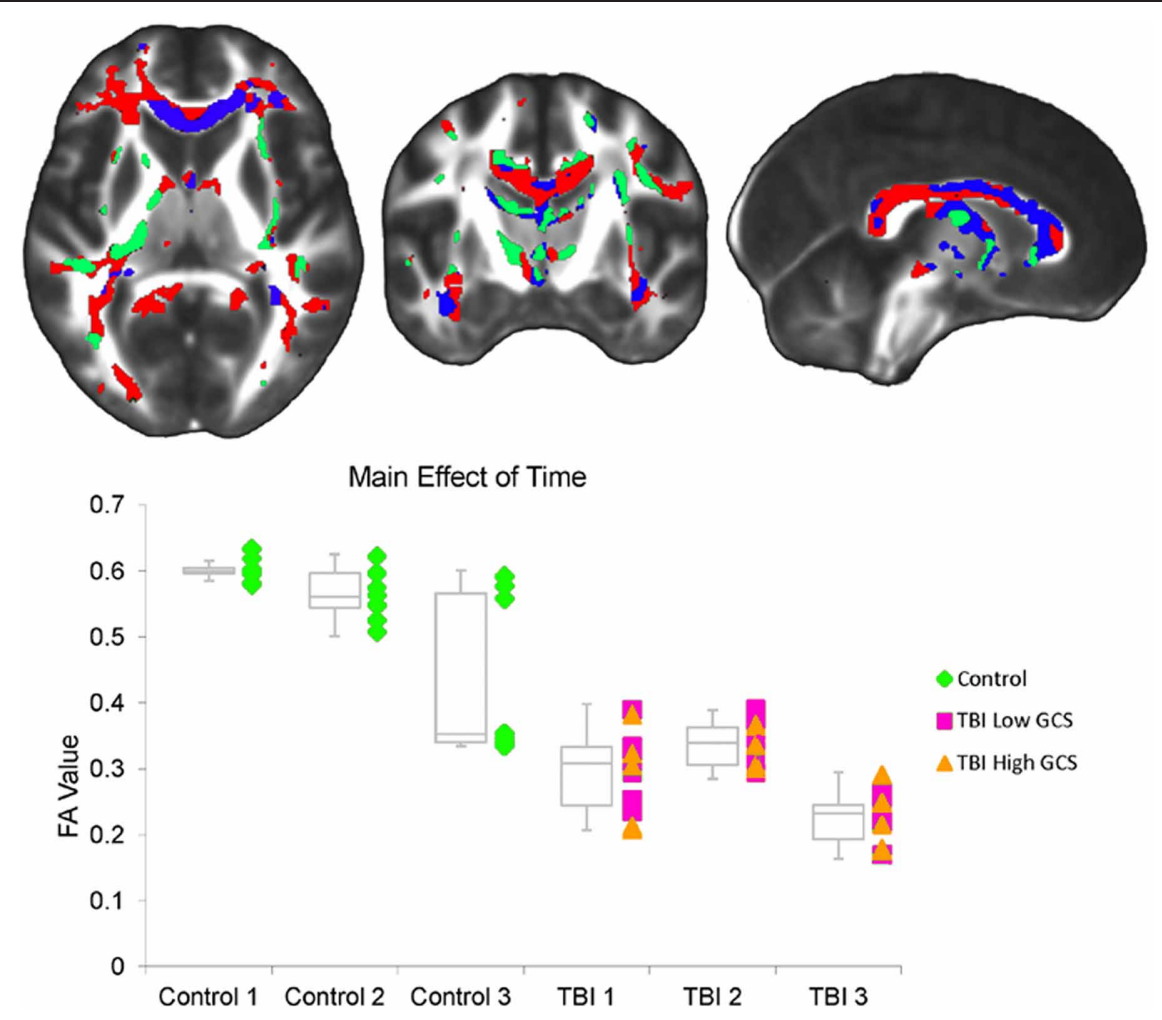

FIGURE 4 | (Top) A significant effect of group was observed within the FA factorial model in several white matter tracts throughout the brain, including the cerebral peduncle, inferior and superior longitudinal fascicule (ILF and SLF), internal and external capsule, inferior fronto-occipital fasciculus, sagittal stratum, corpus callosum, fornix, optic radiations, thalamic radiations, uncinate fasciculus, and corona radiata (red). Axial and radial diffusivity analyses were restricted to regions demonstrating an FA effect. Within this set of regions, a significant effect of group was also observed within the radial diffusivity factorial model (overlapping blue regions), as well as the axial diffusivity factorial model (overlapping green yellow regions). Regions in which a significant effect was seen in the FA, radial diffusivity, and axial diffusivity models are shown in green. The left side of the statistical map is patient left and right is patient right. (Bottom) This graph shows the average FA value for each subject at each of the three time points within the regions demonstrating an FA effect. Controls are marked as green diamonds, TBI patients with a $24 \mathrm{~h}$ GCS score of 7 or lower are marked as pink squares, and patients with a $24 \mathrm{~h}$ GCS score of 10 or higher are marked as orange triangles. Box plots indicate the 75th percentile, median, and 25th percentile FA values of each group at each time point, and whiskers indicate 1.5 times inter-quartile ranges. of TBI (Creed et al., 2011) may underlie the observed FA changes in our TBI cohort.

The fact that longitudinal changes in radial (rather than axial) diffusivity were found in the same regions where FA changes were found warrants further attention. In the literature, findings concerning radial diffusivity and TBI tend to be relatively consistent across studies, whereas findings relating to axial diffusivity are inconsistent. Many studies have reported longitudinal increases in radial diffusivity in the absence of changes in axial diffusivity and like in the current study these changes have frequently been localized to the corpus callosum (Mac Donald et al., 2007; Newcombe et al., 2007; Ewing-Cobbs et al., 2008; Tasker et al., 2010). Differences in axial diffusivity, however, have been inconsistent, with some groups finding increases (Sidaros et al., 2008; Tasker et al., 2010), decreases (Li et al., 2011) or no change (Mac Donald et al., 2007) in both the corpus callosum and other regions. These inconsistencies may be due to differences in how long after injury patient scans were obtained. While FA variations may be related to myelin change, axonal change, or differences in directional coherence of fibers (e.g., presence of absence of crossing fibers), changes in axial diffusivity are thought to be associated primarily with axonal changes while changes in radial diffusivity are thought to relate to myelin changes (Song et al., 2002; Alexander et al., 2007; Xie et al., 2010). These findings indicate that progressive FA loss observed among our subjects was likely driven by progressive myelin pathology, possibly due to persistent inflammation (Ramlackhansingh et al., 2011). While progressive FA increases observed could have been driven by improvements in myelin integrity, the removal of axons with damaged myelin by phagocytotic processes likely also contributed to our result.

While it is not plausible to determine why some regions showed FA increases while others showed decreases with imaging data alone, it is nonetheless instructive to speculate on this matter. One likely contributing factor is the specific injuries sustained by this cohort of TBI patients. The corpus callosum is frequently that center of severe damage during TBI, and the subjects in this cohort were no exception. Several subjects' initial radiology notes included mentions of corpus callosal damage, but none mentioned other tracts specifically. It is possible, therefore, 


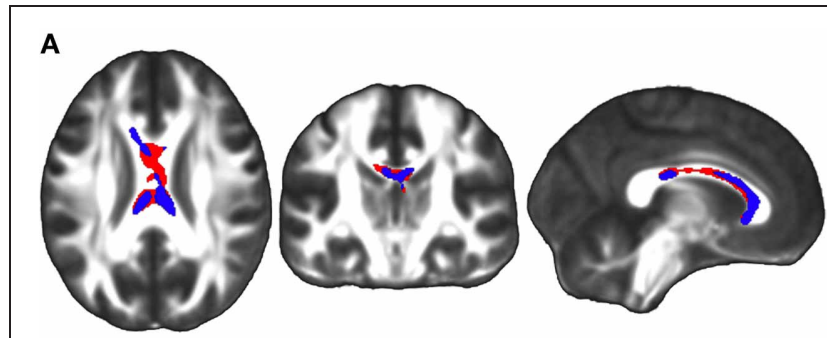

\&

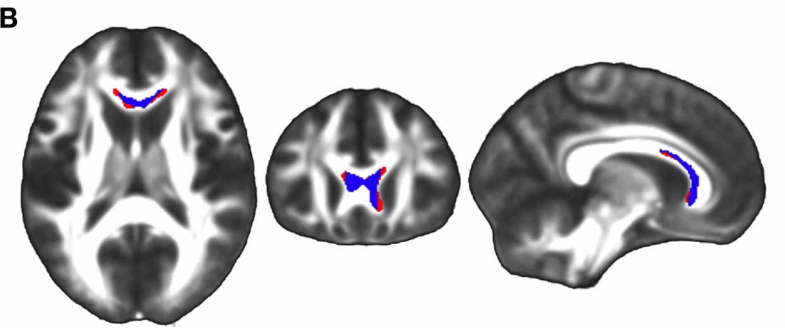

FIGURE 5 | (A) Simple effects analysis within the FA factorial model revealed a significant region of the corpus callosum in which TBI subjects exhibited greater FA at the first time point compared to the third time point (red), indicating a longitudinal FA reduction during the four year study duration. The equivalent test in controls did not produce any significant clusters. Secondary axial and radial diffusivity analyses were renstricted to regions demonstrating an FA effect. Axial and radial diffusivity analyses demonstrated that in this region there was a significant increase in radial diffusivity, but no decrease in axial diffusivity, during the four year study (overlapping blue region). (B) Analysis of this region during the two sub-intervals revealed that there was a significant FA reduction between the first and second time points in the genu of the corpus callosum among TBI subjects (red), but no clusters between the second and third time points. Secondary axial and radial diffusivity analyses were restricted to regions demonstrating an FA effect. Axial and radial diffusivity analyses demonstrated that in this region there was a significant increase in radial diffusivity, but no decrease in axial diffusivity, between the first and second time points (overlapping blue region). The left side of the statistical map is patient left and right is patient right.

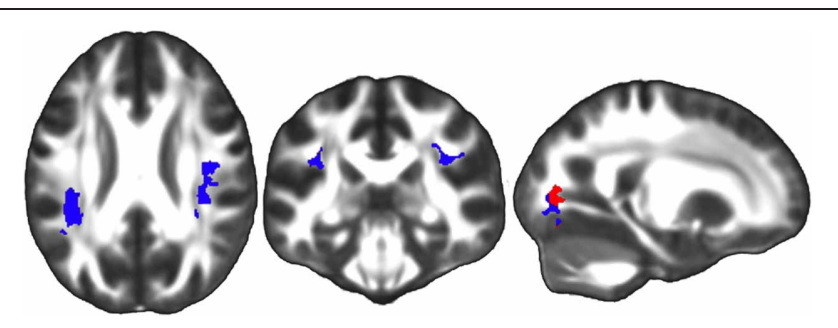

FIGURE 6 | Simple effects analysis within the FA factorial model revealed significant regions in the bilateral SLF in which TBI subjects exhibited greater FA at the third time point compared to the first time point (red), indicating a longitudinal FA increase during the four year study duration. The equivalent test in controls did not produce any significant clusters. Secondary axial and radial diffusivity analyses were restricted to this region demonstrating an FA effect. Axial and radial diffusivity analyses demonstrated that in this region there was a significant decrease in radial diffusivity during the four year study period (overlapping blue region), but no accompanying increase in axial diffusivity. The left side of the statistical map is patient left and right is patient right.

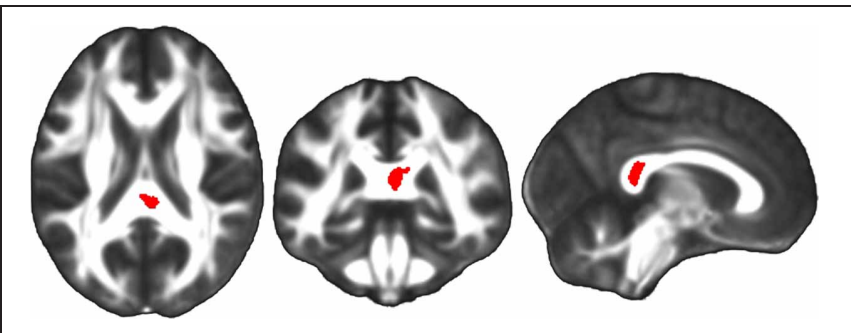

FIGURE 7 | Linear regression analyses, in which neuropsychological test scores were the independent variables and FA maps were the dependent variables, demonstrated a significant correlation between performance on a finger tapping task and FA in the splenium of the corpus callosum among TBI patients at the second time point (red). The left side of the statistical map is patient left and right is patient right.

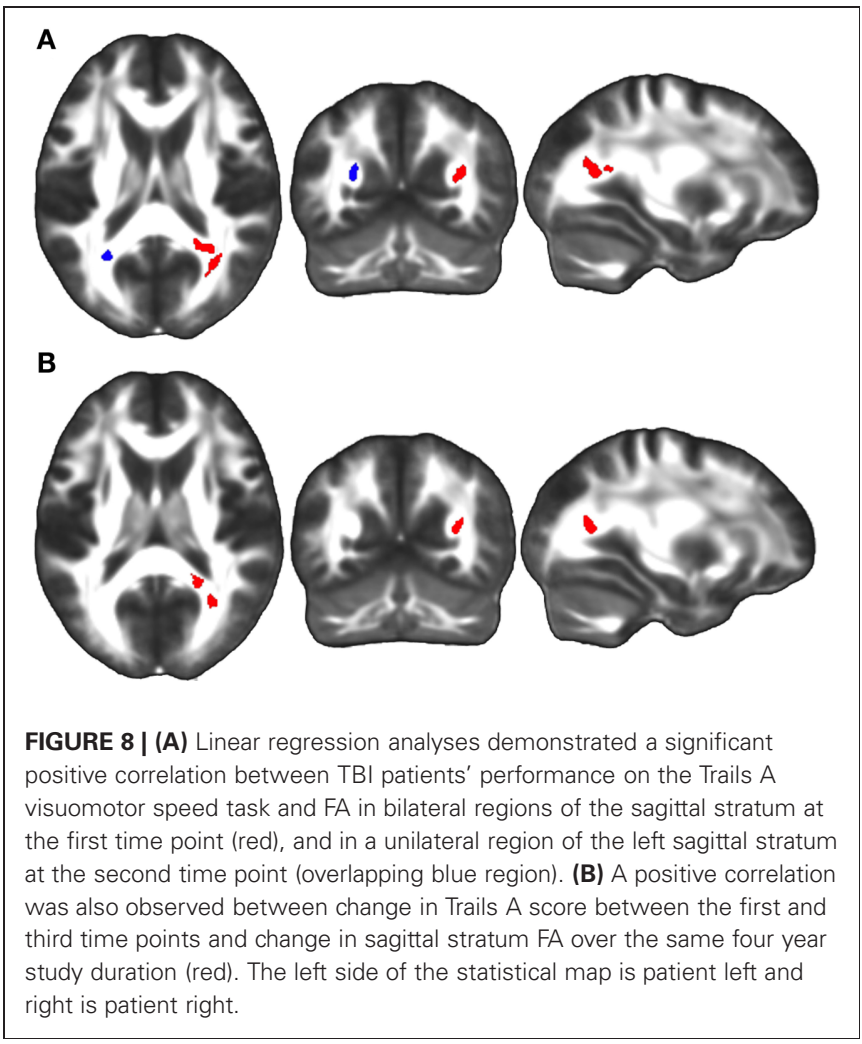

the continued deterioration was observed in regions with greater initial damage. Another complementary possibility is that differential tract properties promote different responses to insult. Research with animal models has demonstrated that astrocytic proliferation can occur in responses to focal injury, but only certain subclasses of neurons are capable of this type of structural remodeling (Blizzard et al., 2011). Further research, most likely in animal models, is necessary to determine why certain regions continue to deteriorate post-injury while others remain static or even improve.

GCS score and WM integrity were not correlated in TBI patients, likely due to the heterogeneity of patients' injuries. It is probable that more severely injured individuals had greater WM 


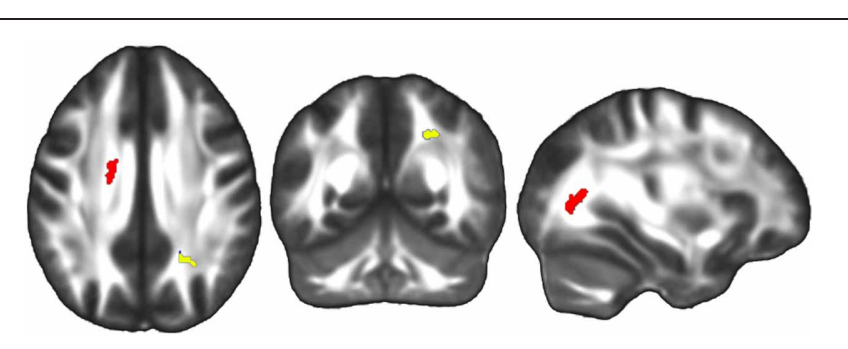

FIGURE 9 | Linear regression analyses revealed a significant positive correlation between change in the cognitive component of the Trails $B$ score between time one and time three and FA change in the left superior SLF and right optic radiation over the same four year study duration (red). Analyses of sub-intervals revealed there was also a significant positive correlation between change in the Trails B cognitive component between the first and second time points, and FA change in the right posterior SLF over the same one year first interval duration (yellow). The left side of the statistical map is patient left and right is patient right.

damage near impact sites, but variable injury locations across subjects precluded group-wise identification of these differences. The neuropsychological correlations we observed were consistent with our hypothesis that FA and neuropsychological task performance would correlate positively. In TBI patients, damage to the splenium indexed by lower FA was associated with slower finger tapping speed, consistent with previous studies linking corpus callosum to manual motor tasks (Muetzel et al., 2008; Caeyenberghs et al., 2011a,b).

Faster performance on the Trails A test was associated with higher FA in the SS, an interesting finding given that this tract is known to be implicated in visuomotor functions. Individuals with greater damage to this tract likely had more difficulty completing the task (Makris et al., 2005; Hao et al., 2011). Over time, this relationship held, with a subset of TBI patients showing longitudinal improvements in Trails A completion speed between the first and third time points and a corresponding increase in FA in the SS over the same interval.

Correlations were also observed between change in the Trails $\mathrm{B}$ cognitive component score over time and change in FA values among TBI patients. A correlation was observed between the first and third time points in the left SLF and the right OR, as well as in the right SLF between the second and third time points. Previous work has demonstrated that FA in the SLF is is associated with complex functions such as attentiveness, working memory and reading skills (Karlsgodt et al., 2008; Frye et al., 2010). The SLF subserves a wide variety of connective functions, and the longitudinal improvements in directional coherence of fibers within this tract among TBI patients in this study may have contributed to improved scores on the Trails $\mathrm{B}$ cognitive component measure. Interestingly, we also found a relationship in the OR (a tract relevant to the relay of visual information from the lateral geniculate nucleus to the visual cortex); this may suggest that subtracting Trails A scores from Trails B scores does not entirely remove the visuomotor element of the task, or possibly that this tract is important for subserving the cognitive component of a visuomotor task. Overall, our neuropsychological correlations demonstrate that the differences in
FA observed in our study do indeed have an impact on cognitive and motor function, and that subtle increases in FA over time reflect WM change that is related to improved functionality in patients. While it should be noted that the neuropsychological correlations reported in the current study may be specific to the set of patients included and their particular patterns of WM damage, it is nonetheless informative to identify functional relevancies of WM change.

In addition to the primary analyses and results presented in this paper, we also conducted an investigatory simple effects analysis of whole-brain radial diffusivity changes within our patients. This analysis was carried out within the radial diffusivity factorial model used in our primary analysis, but the search was not restricted to regions demonstrating FA change. The results of this analysis, which used the same statistical parameters as our primary FA analysis, indicated that within our cohort longitudinal decreases in radial diffusivity (approximating improvements in myelin integrity) were found in regions throughout the brain during the four year study duration. Regions included superior and inferior longitudinal fasiculi, internal and external capsules, the descending corticospinal tract, and forceps major and minor (Figure A1). While this analysis is beyond the planned scope and goals of the current study, it is provocative and may be informative to future research.

The results of this study likely have significant clinical relevance. Specifically, it is notable that we observed WM changes occurring for several years post-injury because the continued malleability of the injured brain holds promise for the effectiveness of treatments well beyond the three to six month window in which treatments are typically prescribed. The FA increase observed in this study has particular relevance to treatment options, as it reflects plasticity and represents a potential physiological basis of rehabilitation. In order to truly assess the relevance of these results to clinical applications, a clinical trial study would be necessary. In such a study, DTI would be used as an outcome measure with the expectation that patients undergoing treatment would exhibit less deterioration or greater improvement in regional brain WM integrity. A positive result would underscore the clinical relevance of our findings.

The current study has methodological limitations that should be considered. Firstly, the results of our study may be limited by methodological limitations imposed by performing a patient and control comparison. For example, it is possible that preprocessing of imaging data and even MR signal of interest can be affected differentially by group. Voxel-wise comparisons of brain images are dependent upon accurate alignment to a template; in order to minimize error, all FA maps were visually inspected for withinsubject tract alignment to the template and alignment across all participants. Another potential limitation concerns the greater ratio of women to men in the control group compared to the TBI group. Due to this, we used gender as a covariate in all analyses. In this study we were not able to account for the intensity or duration of rehabilitations programs in which some of our patients participated. Future clinical trials are critical to understanding how rehabilitation programs impact neurological recovery.

Concurrent volume loss exhibited by TBI patients could also have confounded our results. Volume loss following injury is well 
established in the TBI literature (Levine et al., 2008; Merkley et al., 2008; Sidaros et al., 2009), and has also been observed in this particular cohort of patients (Bendlin et al., 2008). While any DTI study done on this patient population will have results obtained in the context of volume loss, it is nonetheless important to acknowledge that tissue contraction, in addition to microstructural reorganization, may contribute to observed changes in DTI metrics. To ensure that our results were not due solely to volumetric changes, we conducted a native space region of interest analysis on the genu of the corpus callosum. The genu was selected because it is a functionally relevant region and is easily identifiable. FA values were extracted from $3 \mathrm{~mm}$ spherical ROIs placed in the center of the genu in each participant's native space DTI images from Visits 1, 2, and 3. Independent samples twotailed $t$-tests showed that TBI patients had reduced FA compared to controls at all three time points (Visit 1: Controls- $m=0.77$, TBIs- $m=0.67, p=0.005$; Visit 2 : Controls- $m=0.79$, TBIs$m=0.63, p=0.0003$; Visit 3: Controls $-m=0.79$, TBIs- $m=$ $0.61, p=0.001)$. Paired samples two-tailed $t$-tests showed that TBI patients demonstrated a reduction in FA between Visits 1 and $2(p=0.01)$ as well as between visits 1 and $3(p=0.04)$. Paired samples $t$-tests showed no longitudinal changes within the control group. These results mirror the observations made in our whole-brain analysis, and partially allay concerns about the confounding effects of concurrent volume change.

Finally, the results of this study may be limited by the small number of participants that were followed through all three time points. The TBI population and college aged controls are both itinerant populations, and therefore, difficult to track for long periods of time. Notwithstanding this limitation, follow-up of TBI patients over three time points makes this an extremely valuable sample. Furthermore, we did not find significant differences in age, education or injury severity within either group between those who dropped out of the study and those who completed all three visits, suggesting that selective drop-out did not bias the results.

In this study, we show that TBI patients exhibit longitudinal WM changes that continue for at least four years post-injury.

\section{REFERENCES}

Alexander, A. L., Lee, J. E., Lazar, M., and Field, A. S. (2007). Diffusion tensor imaging of the brain. Neurotherapeutics 4, 316-329.

Anderson, C. V., Bigler, E. D., and Blatter, D. D. (1995). Frontal lobe lesions, diffuse damage, and neuropsychological functioning in traumatic brain-injured patients. J. Clin. Exp. Neuropsychol. 17, 900-908.

Arfanakis, K., Haughton, V. M., Carew, J. D., Rogers, B. P., Dempsey, R. J., and Meyerand, M. E. (2002). Diffusion tensor MR imaging in diffuse axonal injury. AJNR Am. J. Neuroradiol. 23, 794-802.

Bendlin, B. B., Ries, M. L., Lazar, M., Alexander, A. L., Dempsey, R. J., Rowley, H. A., Sherman, J. E., and

These changes include both progressive reductions in FA (observed in the corpus callosum) as well as progressive FA increases (observed in the bilateral SLF). Within the regions where FA changes were found, radial diffusivity alterations were present-suggesting myelin changes continued to occur throughout the period studied. Furthermore, neuropsychological correlations indicate that diffusion metrics are related to cognitive and motor abilities, and that improved directional coherence within tracts is relevant to improved task performance in certain brain regions among TBI patients. The fact that changes continued to develop for several years post-injury provides support for the hypothesis that TBI is not merely an event but rather the initiation of a prolonged disease state with potentially lifelong systemic impacts. FA increases are of particular note due to the unprecedented nature of these findings. TBI is generally conceptualized as strictly a degenerative condition and evidence of what might be long-term neurological improvement could be of significant clinical importance. We strive to continue to improve our understanding of these long-term effects following traumatic injury. An important area of work going forward will be to capitalize on signs of gradual long-term neurological and functional improvement observed in TBI, and to further understand the brain correlates of ameliorative change, which likely occurs alongside progressive degeneration.

\section{ACKNOWLEDGMENTS}

This study was supported by a Merit Review Grant from the Department of Veterans Affairs, the NIH MH65723 (SCJ), NIH AG000213 and by the facilities and resources of the William S. Middleton Memorial Veterans Hospital. The assistance of Lisa Newman, Amy Hawley, Donald McLaren, and Erik Kastman is greatly appreciated. We would also like to acknowledge the support of researchers and staff at the Waisman Center, University of Wisconsin, Madison, where MR imaging took place. Finally, we thank all the patients who took part in this study. The contents of this report do not necessarily represent the views of the Department of Veterans Affairs or the United States Government.

dendritic structural plasticity. Cereb. Cortex 21, 281-291.

Bombardier, C. H., Fann, J. R., Temkin, N. R., Esselman, P. C., Barber, J., and Dikmen, S. S. (2010). Rates of major depressive disorder and clinical outcomes following traumatic brain injury. JAMA 303 , 1938-1945.

Caeyenberghs, K., Leemans, A., Coxon, J., Leunissen, I., Drijkoningen, D., Geurts, M., Gooijers, J., Michiels, K., Sunaert, S., and Swinnen, S. P. (2011a). Bimanual coordination and corpus callosum microstructure in young adults with traumatic brain injury: a diffusion tensor imaging study. J. Neurotrauma 28, 897-913.

Caeyenberghs, K., Leemans, A., Geurts, M., Linden, C. V., Smits-Engelsman,
B. C., Sunaert, S., and Swinnen, S. P. (2011b). Correlations between white matter integrity and motor function in traumatic brain injury patients. Neurorehabil. Neural Repair 25, 492-502.

Chan, J. H., Tsui, E. Y., Peh, W. C., Fong, D., Fok, K. F., Leung, K. M., Yuen, M. K., and Fung, K. K. (2003). Diffuse axonal injury: detection of changes in anisotropy of water diffusion by diffusion-weighted imaging. Neuroradiology 45, 34-38.

Creed, J. A., DiLeonardi, A. M., Fox, D. P., Tessler, A. R., and Raghupathi, R. (2011). Concussive brain trauma in the mouse results in acute cognitive deficits and sustained impairment of axonal function. J. Neurotrauma 28, 547-563. 
Ewing-Cobbs, L., Prasad, M. R., Swank, P., Kramer, L., Cox, C. S. Jr., Fletcher, J. M., Barnes, M., Zhang, X., and Hasan, K. M. (2008). Arrested development and disrupted callosal microstructure following pediatric traumatic brain injury: relation to neurobehavioral outcomes. Neuroimage 42, 1305-1315.

Field, A. S., Hasan, K., Jellison, B. J., Arfanakis, K., and Alexander, A. L. (2003). Diffusion tensor imaging in an infant with traumatic brain swelling. AJNR Am. J. Neuroradiol. 24, 1461-1464.

Filippi, C. G., Ulug, A. M., Ryan, E., Ferrando, S. J., and van Gorp, W. (2001). Diffusion tensor imaging of patients with HIV and normal-appearing white matter on MR images of the brain. AJNR Am. J. Neuroradiol. 22, 277-283.

Forman, S. D., Cohen, J. D., Fitzgerald, M., Eddy, W. F., Mintun, M. A., and Noll, D. C. (1995). Improved assessment of significant activation in functional magnetic resonance imaging (fMRI): use of a clustersize threshold. Magn. Reson. Med. 33, 636-647.

Frye, R. E., Hasan, K., Malmberg, B., Desouza, L., Swank, P., Smith, K., and Landry, S. (2010). Superior longitudinal fasciculus and cognitive dysfunction in adolescents born preterm and at term. Dev. Med. Child Neurol. 52, 760-766.

Gale, S. D., Johnson, S. C., Bigler, E. D., and Blatter, D. D. (1995). Nonspecific white matter degeneration following traumatic brain injury. J. Int. Neuropsychol. Soc. 1, 17-28.

Hao, X., Xu, D., Bansal, R., Dong, Z., Liu, J., Wang, Z., Kangarlu, A., Liu, F., Duan, Y., Shova, S., Gerber, A. J., and Peterson, B. S. (2011). Multimodal magnetic resonance imaging: the coordinated use of multiple, mutually informative probes to understand brain structure and function. Hum. Brain Mapp. doi: 10.1002/hbm.21440. [Epub ahead of print].

Karlsgodt, K. H., van Erp, T. G., Poldrack, R. A., Bearden, C. E., Nuechterlein, K. H., and Cannon, T. D. (2008). Diffusion tensor imaging of the superior longitudinal fasciculus and working memory in recentonset schizophrenia. Biol. Psychiatry 63, 512-518.

Kim, J., Avants, B., Patel, S., Whyte, J., Coslett, B. H., Pluta, J., Detre, J. A., and Gee, J. C. (2008). Structural consequences of diffuse traumatic brain injury: a large deformation tensor-based morphometry study. Neuroimage 39, 1014-1026.

Kiraly, M., and Kiraly, S. J. (2007). Traumatic brain injury and delayed sequelae: a review-traumatic brain injury and mild traumatic brain injury (concussion) are precursors to later-onset brain disorders, including early-onset dementia. ScientificWorldJournal 7, 1768-1776.

Kraus, M. F., Susmaras, T., Caughlin, B. P., Walker, C. J., Sweeney, J. A., and Little, D. M. (2007). White matter integrity and cognition in chronic traumatic brain injury: a diffusion tensor imaging study. Brain $130(\mathrm{Pt}$ 10), 2508-2519.

Kumar, R., Saksena, S., Husain, M., Srivastava, A., Rathore, R. K., Agarwal, S., and Gupta, R. K. (2010). Serial changes in diffusion tensor imaging metrics of corpus callosum in moderate traumatic brain injury patients and their correlation with neuropsychometric tests: a 2-year follow-up study. J. Head Trauma Rehabil. 25, 31-42.

Le Bihan, D. (1991). Molecular diffusion nuclear magnetic resonance imaging. Magn. Reson. Q. 7, 1-30.

Levin, H. S. (2003). Neuroplasticity following non-penetrating traumatic brain injury. Brain Inj. 17, 665-674.

Levine, B., Kovacevic, N., Nica, E. I., Cheung, G., Gao, F., Schwartz, M. L., and Black, S. E. (2008). The Toronto traumatic brain injury study: injury severity and quantified MRI. Neurology 70, 771-778.

Li, J., Li, X. Y., Feng, D. F., and Gu, L. (2011). Quantitative evaluation of microscopic injury with diffusion tensor imaging in a rat model of diffuse axonal injury. Eur. J. Neurosci. 33, 933-945.

Lin, M. R., Chiu, W. T., Chen, Y. J., Yu, W. Y., Huang, S. J., and Tsai, M. D. (2010). Longitudinal changes in the health-related quality of life during the first year after traumatic brain injury. Arch. Phys. Med. Rehabil. 91, 474-480.

Ljungqvist, J., Nilsson, D., Ljungberg, M., Sorbo, A., Esbjornsson, E., Eriksson-Ritzen, C., and Skoglund, T. (2011). Longitudinal study of the diffusion tensor imaging properties of the corpus callosum in acute and chronic diffuse axonal injury. Brain Inj. 25, 370-378.

Mac Donald, C. L., Dikranian, K., Bayly, P., Holtzman, D., and Brody, D. (2007). Diffusion tensor imaging reliably detects experimental traumatic axonal injury and indicates approximate time of injury. J. Neurosci. 27, 11869-11876.
Makris, N., Kennedy, D. N., McInerney, S., Sorensen, A. G., Wang, R. Caviness, V. S. Jr., and Pandya, D. N. (2005). Segmentation of subcomponents within the superior longitudinal fascicle in humans: a quantitative, in vivo, DT-MRI study. Cereb. Cortex 15, 854-869.

Malec, J. F., Brown, A. W., Moessner, A. M., Stump, T. E., and Monahan, P. (2010). A preliminary model for posttraumatic brain injury depression. Arch. Phys. Med. Rehabil. 91, 1087-1097.

Marquez de la Plata, C. D., Hart, T., Hammond, F. M., Frol, A. B., Hudak, A., Harper, C. R., O’NeilPirozzi, T. M., Whyte, J., Carlile, M., and Diaz-Arrastia, R. (2008) Impact of age on long-term recovery from traumatic brain injury. Arch. Phys. Med. Rehabil. 89, 896-903.

Masel, B. E., and DeWitt, D. S. (2010). Traumatic brain injury: a disease process, not an event. J. Neurotrauma 27, 1529-1540.

Matsukawa, H., Shinoda, M., Fujii, M., Takahashi, O., Yamamoto, D., Murakata, A., and Ishikawa, R. (2011). Genu of corpus callosum in diffuse axonal injury induces a worse 1-year outcome in patients with traumatic brain injury. Acta Neurochir. (Wien) 153, 1687-1693. discussion 1693-1694.

Merkley, T. L., Bigler, E. D., Wilde, E. A., McCauley, S. R., Hunter, J. V., and Levin, H. S. (2008). Diffuse changes in cortical thickness in pediatric moderate-to-severe traumatic brain injury. J. Neurotrauma 25, 1343-1345.

Muetzel, R. L., Collins, P. F., Mueller, B. A. M., Schissel, A., Lim, K. O., and Luciana, M. (2008). The development of corpus callosum microstructure and associations with bimanual task performance in healthy adolescents. Neuroimage 39, 1918-1925.

Nakayama, N., Okumura, A., Shinoda, J., Yasokawa, Y. T., Miwa, K., Yoshimura, S. I., and Iwama, T. (2006). Evidence for white matter disruption in traumatic brain injury without macroscopic lesions. J. Neurol. Neurosurg. Psychiatry 77, 850-855.

Newcombe, V. F., Williams, G. B., Nortje, J., Bradley, P. G., Harding, S. G., Smielewski, P., Coles, J. P., Maiya, B., Gillard, J. H., Hutchinson, P. J., Pickard, J. D. Carpenter, T. A., and Menon, D. K. (2007). Analysis of acute traumatic axonal injury using diffusion tensor imaging. Br. J. Neurosurg. 21, 340-348.
Povlishock, J. T. (2000). Pathophysiology of neural injury: therapeutic opportunities and challenges. Clin. Neurosurg. 46, 113-126.

Povlishock, J. T., and Christman, C. W. (1995). The pathobiology of traumatically induced axonal injury in animals and humans: a review of current thoughts. J. Neurotrauma 12, 555-564.

Ramlackhansingh, A. F., Brooks, D. J., Greenwood, R. J., Bose, S. K., Turkheimer, F. E., Kinnunen, K. M., Gentleman, S., Heckemann, R. A., Gunanayagam, K., Gelosa, G., and Sharp, D. J. (2011). Inflammation after trauma: microglial activation and traumatic brain injury. Ann. Neurol. 70, 374-383.

Risdall, J. E., and Menon, D. K. (2010). Traumatic brain injury. Philos. Trans. R. Soc. Lond. B Biol. Sci. 366, 241-250.

Rubovitch, V., Ten-Bosch, M., Zohar, O., Harrison, C. R., Tempel-Brami, C., Stein, E., Hoffer, B. J., Balaban, C. D., Schreiber, S., Chiu, W. T., and Pick, C. G. (2011). A mouse model of blast-induced mild traumatic brain injury. Exp. Neurol. 232, 280-289.

Sharp, D. J., and Ham, T. E. (2011). Investigating white matter injury after mild traumatic brain injury. Curr. Opin. Neurol. 24, 558-563.

Sidaros, A., Engberg, A. W., Sidaros, K., Liptrot, M. G., Herning, M., Petersen, P., Paulson, O. B., Jernigan, T. L., and Rostrup, E. (2008). Diffusion tensor imaging during recovery from severe traumatic brain injury and relation to clinical outcome: a longitudinal study. Brain 131(Pt 2), 559-572.

Sidaros, A., Skimminge, A., Liptrot, M. G., Sidaros, K., Engberg, A. W., Herning, M., Paulson, O. B., Jernigan, T. L., and Rostrup, E. (2009). Long-term global and regional brain volume changes following severe traumatic brain injury: a longitudinal study with clinical correlates. Neuroimage 44, 1-8.

Smith, S. M., Jenkinson, M., Woolrich, M. W., Beckmann, C. F., Behrens, T. E., Johansen-Berg, H., Bannister, P. R., De Luca, M., Drobnjak, I., Flitney, D. E., Niazy, R. K., Saunders, J., Vickers, J., Zhang, Y., De Stefano, N., Brady, J. M., and Matthews, P. M. (2004). Advances in functional and structural MR image analysis and implementation as FSL. Neuroimage 23(Suppl. 1), S208-S219.

Song, S. K., Sun, S. W., Ju, W. K., Lin, S. J., Cross, A. H., and Neufeld, A. H. (2003). Diffusion 
tensor imaging detects and differentiates axon and myelin degeneration in mouse optic nerve after retinal ischemia. Neuroimage 20, 1714-1722.

Song, S. K., Sun, S. W., Ramsbottom, M. J., Chang, C., Russell, J., and Cross, A. H. (2002). Dysmyelination revealed through MRI as increased radial (but unchanged axial) diffusion of water. Neuroimage 17, 1429-1436.

Staudt, M. (2010). Brain plasticity following early life brain injury: insights from neuroimaging. Semin. Perinatol. 34, 87-92.

Tasker, R. C., Westland, A. G., White, D. K., and Williams, G. B. (2010). Corpus callosum and inferior forebrain white matter microstructure are related to functional outcome from raised intracranial pressure in child traumatic brain injury. Dev. Neurosci. 32, 374-384.

Trivedi, M. A., Ward, M. A., Hess, T. M., Gale, S. D., Dempsey, R. J.,
Rowley, H. A., and Johnson, S. C. (2007). Longitudinal changes in global brain volume between 79 and 409 days after traumatic brain injury: relationship with duration of coma. J. Neurotrauma 24, 766-771.

Wang, J. Y., Bakhadirov, K., Abdi, H., Devous, M. D. Sr., Marquez de la Plata, C. D., Moore, C., Madden, C. J., and Diaz-Arrastia, R. (2011). Longitudinal changes of structural connectivity in traumatic axonal injury. Neurology 77, 818-826.

Wheeler-Kingshott, C. A., and Cercignani, M. (2009). About "axial" and "radial" diffusivities. Magn. Reson. Med. 61, 1255-1260.

Wu, T. C., Wilde, E. A., Bigler, E. D., Li, X., Merkley, T. L., Yallampalli, R., McCauley, S. R., Schnelle, K. P., Vasquez, A. C., Chu, Z., Hanten, G., Hunter, J. V., and Levin, H. S. (2010). Longitudinal changes in the corpus callosum following pediatric traumatic brain injury. Dev. Neurosci. 32, 361-373.

Xie, M., Tobin, J. E., Budde, M. D., Chen, C. I., Trinkaus, K., Cross, A. H., McDaniel, D. P., Song, S. K., and Armstrong, R. C. (2010). Rostrocaudal analysis of corpus callosum demyelination and axon damage across disease stages refines diffusion tensor imaging correlations with pathological features. J. Neuropathol. Exp. Neurol. 69, 704-716.

$\mathrm{Xu}$, J., Rasmussen, I. A., Lagopoulos, J., and Haberg, A. (2007). Diffuse axonal injury in severe traumatic brain injury visualized using high-resolution diffusion tensor imaging. J. Neurotrauma 24 , 753-765.

Conflict of Interest Statement: The authors declare that the research was conducted in the absence of any commercial or financial relationships that could be construed as a potential conflict of interest.

Received: 07 February 2012; accepted: 20 May 2012; published online: 19 June 2012.

Citation: Farbota KD, Bendlin BB, Alexander AL, Rowley HA, Dempsey RJ and Johnson SC (2012) Longitudinal diffusion tensor imaging and neuropsychological correlates in traumatic brain injury patients. Front. Hum. Neurosci. 6:160. doi: 10.3389/fnhum. 2012.00160

Copyright (c) 2012 Farbota, Bendlin, Alexander, Rowley, Dempsey and Johnson. This is an open-access article distributed under the terms of the Creative Commons Attribution Non Commercial License, which permits non-commercial use, distribution, and reproduction in other forums, provided the original authors and source are credited. 


\section{APPENDIX}

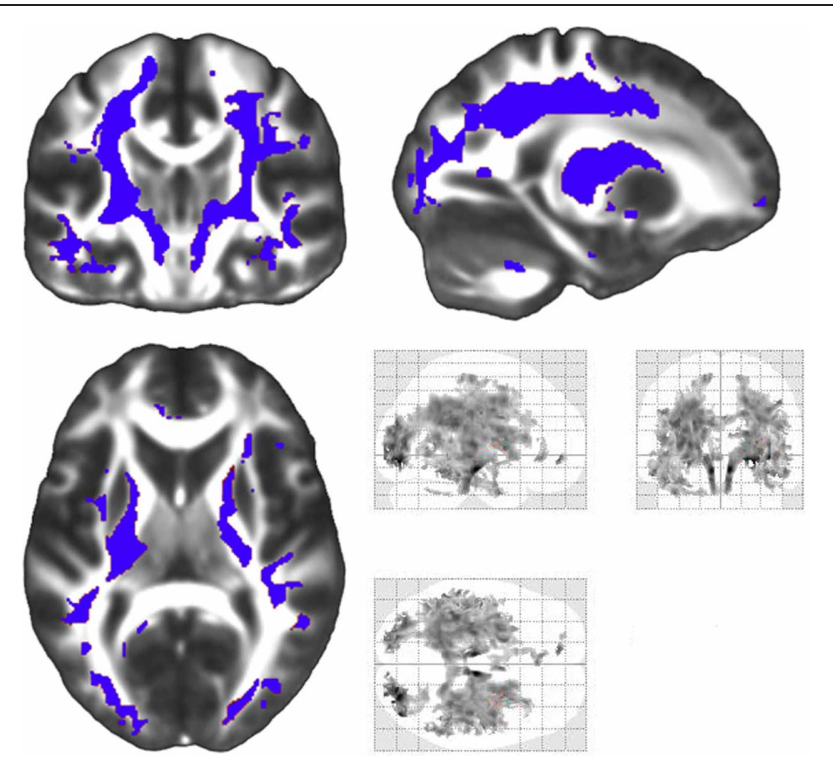

FIGURE A1 | A supplementary whole-brain analysis of radial diffusivity change among patients that was not restricted to regions

demonstrating an FA effect was conducted. This analysis was carried out in the same radial diffusivity factorial model used in the restricted radial diffusivity analyses, and statistical thresholds used in FA analyses were employed. This analysis examined decreases in radial diffusivity during the four year study duration. Regions of decreased radial diffusivity included significant longitudinal decreases in radial diffusivity in superior and inferior longitudinal fasiculi, internal and external capsules, forceps major and minor and the descending corticospinal tract (blue). The tubular regions of change that extend through the descending corticospinal tract were not constrained on any side by the white matter mask employed in our analyses. The left side of the statistical map is patient left and right is patient right. 